\title{
Specific survivin dual fluorescence resonance energy transfer molecular beacons for detection of human bladder cancer cells
}

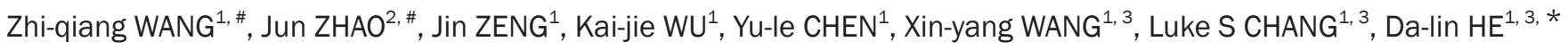

${ }^{1}$ Department of Urology, the First Affiliated Hospital of the Medical School of Xi-an Jiaotong University, Xi-an 710061, China; ${ }^{2}$ Department of Urology, the Affiliated Hospital of Qingdao University Medical College, Qingdao 266003, China; ${ }^{3}$ Oncological Research Lab, Key Laboratory of Environment and Genes Related to Diseases, Ministry of Education, Xi-an Jiaotong University School of Medicine, Xi-an 710061, China

Aim: Survivin molecular beacons can be used to detect bladder cancer cells in urine samples non-invasively. The aim of this study is to improve the specificity of detection of bladder cancer cells using survivin dual fluorescence resonance energy transfer molecular beacons (FRET MBs) that have fluorophores forming one donor-acceptor pair.

Methods: Survivin-targeting dual fluorescence resonance energy transfer molecular beacons with unique target sequences were designed, which had no overlap with the other genes in the apoptosis inhibitor protein family. Human bladder cancer cell lines 5637 , 253J and T24, as well as the exfoliated cells in the urine of healthy adults and patients with bladder cancer were examined. Images of cells were taken using a laser scanning confocal fluorescence microscope. For assays using dual FRET MBs, the excitation wavelength was $488 \mathrm{~nm}$, and the emission detection wavelengths were $520 \pm 20 \mathrm{~nm}$ and $560 \pm 20 \mathrm{~nm}$, respectively.

Results: The human bladder cancer cell lines and exfoliated cells in the urine of patients with bladder cancer incubated with the survivin dual FRET MBs exhibited strong fluorescence signals. In contrast, no fluorescence was detected in the survivin-negative human dermal fibroblasts-adult (HDF-a) cells or exfoliated cells in the urine of healthy adults incubated with the survivin dual FRET MBs.

Conclusion: The results suggest that the survivin dual FRET MBs may be used as a specific and non-invasive method for early detection and follow-up of patients with bladder cancer.

Keywords: molecular beacons; fluorescence resonance energy transfer; survivin; human bladder cancer

Acta Pharmacologica Sinica (2011) 32: 1522-1528; doi: 10.1038/aps.2011.122; published online 24 Oct 2011

\section{Introduction}

Urinary bladder cancer $(\mathrm{BCa})$ is a common malignancy ${ }^{[1,2]}$, with the highest recurrence rate of all cancers. The recurrence rate ranges from $50 \%$ to $70 \%$ and the progression rate to muscle-invasive disease from $10 \%$ to $15 \%{ }^{[3]}$. Early detection of $\mathrm{BCa}$ and its recurrence is essential for improved prognosis and long-term survival ${ }^{[4]}$. Therefore, much research has been directed toward identifying the best possible non-invasive, urine-based assays for detection, surveillance and prediction of recurrence and/or progression ${ }^{[4,5]}$. However, urine markers have not been used in early detection or screening for $\mathrm{BCa}$ to

\footnotetext{
\# These authors contributed equally to the work.

* To whom correspondence should be addressed.

E-mail dalinhexjtu@126.com

Received 2011-04-10 Accepted 2011-08-09
}

date $^{[6]}$.

Previous studies showed that survivin molecular beacons (MBs) provided a sensitive and convenient method to detect BCa cells in urine samples ${ }^{[7,8]}$. However, conventional MBs can be degraded by nucleases or opened by nucleic acid binding proteins, leading to false positive signals ${ }^{[9]}$. Here, we tried to improve the specificity of detection of BCa using survivin dual fluorescence resonance energy transfer (FRET) MBs whose fluorophores formed one donor-acceptor pair. Each MB pair used sequences complementary to adjacent regions on the same oligonucleotide target so that FRET occurred only when both beacons were hybridized to the target (Figure 1). The dual FRET MBs have been used successfully for mRNA detection in living cells and for monitoring changes in mRNA levels and mRNA cell localization ${ }^{[10,11]}$. However, this approach requires a complex permeabilization method for 
A
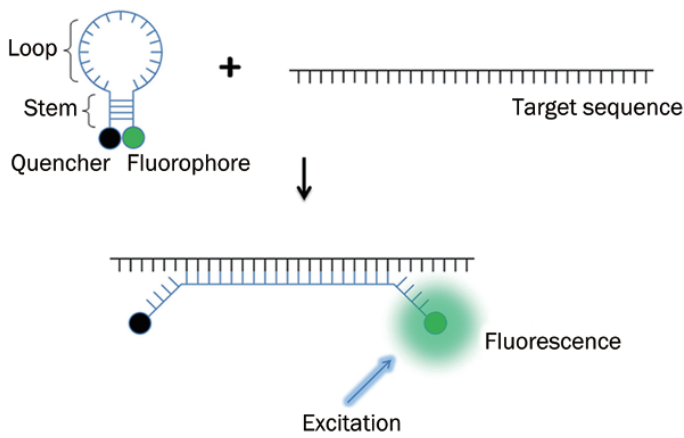

B

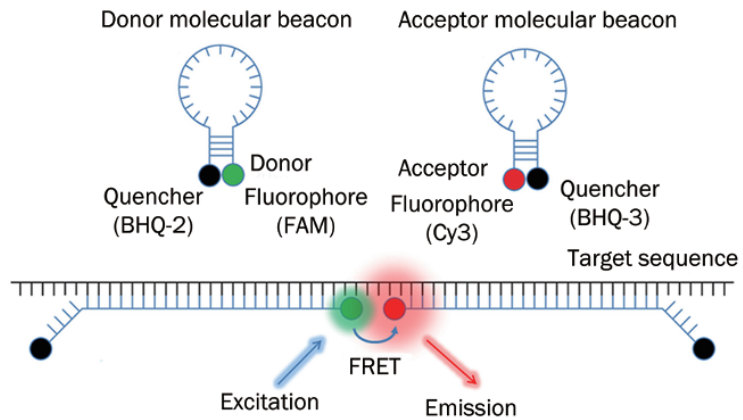

Figure 1. A schematic illustration showing the working mechanism of an $M B$ and a dual FRET MB. (A) The MB adopts a stem-loop structure and thus holds the fluorophore (green) and quencher (black) in close proximity. As a result, the fluorescence emission of the fluorophore is strongly suppressed (in the absence of a target). The target sequence hybridizes with the loop domain of the MB and forces the stem helix to open, whereupon fluorescence is restored because of the spatial separation of the fluorophore from the quencher. (B) Hybridization of donor and acceptor MBs to adjacent regions on the same mRNA target results in FRET between donor fluorophores (FAM, excitation at $490 \mathrm{~nm}$ and emission at $520 \mathrm{~nm}$ ) and acceptor fluorophores (Cy3, excitation at 550 $\mathrm{nm}$ and emission at $570 \mathrm{~nm}$ ) upon donor excitation (490 nm). Because only the donor fluorophore is directly excited, the emission detected from the acceptor fluorophore is due to FRET.

cellular delivery and thus has been hard to popularize in clinical practice ${ }^{[11]}$. In our previous study, we showed that fixing cells with ice-cold acetone was a simple, effective method to use with $\mathrm{MBs}^{[8,12]}$. In this study, we aimed to use this simple method to determine whether dual FRET MBs could be used to detect survivin mRNA in BCa cells, which might lay the foundation for clinical applications.

\section{Materials and methods}

Survivin dual FRET MBs: design and synthesis

The survivin-targeting MBs were designed so that the target sequences were unique, having no overlap with other genes in the inhibitor of apoptosis protein (IAP) family ${ }^{[11]}$. As shown in Table 1, we designed two pairs of dual FRET MBs (complementary to target sequences) and one pair of random dual MBs (non-complementary to target sequences). The probe lengths of survivin-targeting donor and acceptor MBs were 15 and 16 bases, respectively. The 'random' beacons had a probe length of 16 bases. All MBs had the shared-stem design, with a stem length of five bases; they had an unmodified oligonucleotide backbone. The survivin MBs and random beacons were synthesized by Invitrogen (Shanghai, China). All of the synthetic targets were synthesized by Sangon Biological Engineering Technology (Shanghai, China).

\section{Solution assays of probe-target hybridization}

All solution studies of the probe-target hybridizations were carried out in PBS without calcium and magnesium. After incubation of the probes and targets at $37^{\circ} \mathrm{C}$ for $60 \mathrm{~min}$, fluorescence intensity was measured by time-resolved fluorescence spectroscopy (QM4, PTI, Inc, Birmingham, NJ, USA), with $515 \mathrm{~nm} \mathrm{Cy3}$ (donor fluorophore) second peak excitation and 530-800 nm emission wavelength detection, and $490 \mathrm{~nm}$ FAM (donor fluorophore) excitation and 500-650 $\mathrm{nm}$ emission wavelength detection. Concentrations of $200 \mathrm{nmol} / \mathrm{L}$ donor, $200 \mathrm{nmol} / \mathrm{L}$ acceptor MBs and $200 \mathrm{nmol} / \mathrm{L}$ targets were used in a total volume of $2 \mathrm{~mL}$.

\section{Cell culture and glass coverslip preparation}

The human BCa cell line 5637 (obtained from ATCC) was maintained at $37^{\circ} \mathrm{C}, 5 \% \mathrm{CO}_{2}$ in RPMI-1640 (ScienCell Laboratory, USA) medium supplemented with $10 \%$ fetal bovine serum (FBS). The human BCa cell lines 253J and T24 (obtained from ATCC) were maintained at $37^{\circ} \mathrm{C}, 5 \% \mathrm{CO}_{2}$ in Dulbecco's

Table 1. Target sequences and the design of molecular beacons.

Gene

Survivin
Sequences

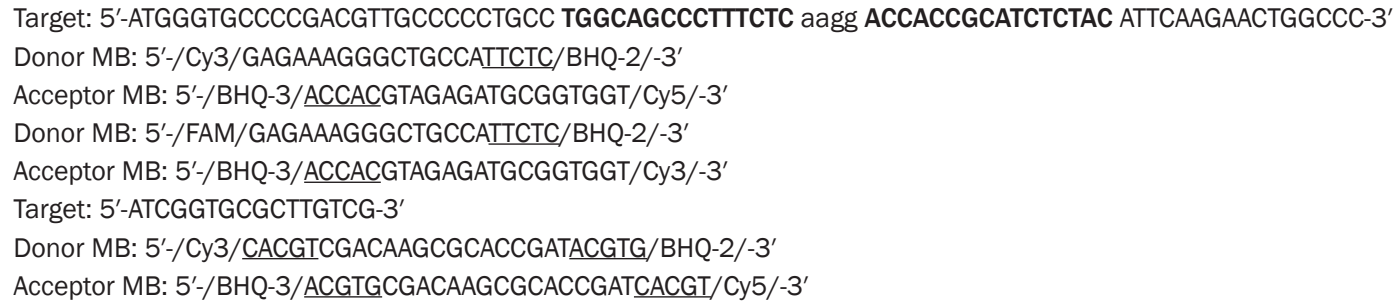

Molecular beacon (MB): underlined bases, bases added to create the stem domain. Target: lowercase bold, bases between two target sequences of the donor and acceptor beacon ${ }^{[11]}$. 
modified Eagle medium (DMEM, ScienCell Laboratory, USA) supplemented with 10\% FBS. Normal human dermal fibroblasts-adult (HDF-a, ScienCell Laboratory, USA) were grown in fibroblast medium (ScienCell Laboratory, USA) supplemented with $2 \%$ FBS, fibroblast growth supplement and penicillin/streptomycin solution. The 5637, 253J, T24, and HDF-a cells were cultured on glass coverslips for $24-48 \mathrm{~h}$. The coverslips, which had cells adhering to them, were then fixed with ice-cold acetone for 5 to $10 \mathrm{~min}$, air-dried and incubated with survivin dual FRET MBs (concentrations of $200 \mathrm{nmol} / \mathrm{L}$ donor and $200 \mathrm{nmol} / \mathrm{L}$ acceptor MBs) at $37^{\circ} \mathrm{C}$ for $60 \mathrm{~min}$ in a dark, humid chamber before being examined by laser scanning confocal fluorescence microscopy or fluorescence microscopy.

\section{Western blot analysis}

Western blot analysis was performed as previously described $^{[13]}$. Human BCa cell lines and HDF-a cells were washed with cold PBS twice; then, total cellular protein lysates were prepared with RIPA buffer $[50 \mathrm{mmol} / \mathrm{L}$ Tris $(\mathrm{pH} 8.0)$, $150 \mathrm{mmol} / \mathrm{L} \mathrm{NaCl}, 0.1 \%$ SDS, $1 \% \mathrm{NP} 40$ and $0.5 \%$ sodium deoxycholate] containing proteinase inhibitors $(1 \%$ protease inhibitor cocktail and $1 \mathrm{mmol} / \mathrm{L}$ phenylmethanesulfonyl fluoride, both from Sigma, St Louis, MO, USA). A total of $30 \mu \mathrm{g}$ of protein was separated by $12 \%$ SDS-PAGE and transferred to nitrocellulose membranes. The membranes were blocked at room temperature for $1 \mathrm{~h}$ with $5 \%$ milk in Tris-buffered saline with $0.1 \%$ Tween-20 (pH 7.6, TBST). The membranes were incubated with primary antibodies (anti-survivin antibody, Santa Cruz, USA, sc-17779, 1:500 dilution) at room temperature for $2 \mathrm{~h}$. After being washed with TBST, membranes were incubated with secondary antibodies coupled to horseradish peroxidase at room temperature for $1 \mathrm{~h}$ and visualized with an ECL chemiluminescence detection system (Pierce, Rockford, IL, USA). Loading differences were normalized using a monoclonal GAPDH antibody.

\section{Urine sample collection and assay}

Exfoliated cells were obtained from $50 \mathrm{~mL}$ of the second morning voiding of urine from patients with $\mathrm{BCa}$ and from healthy adults. Urine samples were processed immediately after collection. After centrifugation for $10 \mathrm{~min}(2500 \times \mathrm{g})$, the sediment was resuspended in PBS and placed on glass slides with cytospin. The glass slides with adherent cells were fixed with pre-chilled acetone for 5 to $10 \mathrm{~min}$, air-dried and incubated with survivin dual FRET MBs (concentrations of $200 \mathrm{nmol} / \mathrm{L}$ donor and $200 \mathrm{nmol} / \mathrm{L}$ acceptor MBs) at $37^{\circ} \mathrm{C}$ for $60 \mathrm{~min}$ in a dark, humid chamber and then examined with an Olympus fluorescence microscope (Model BX51, Olympus Optical, Tokyo, Japan). Fluorescence images were analyzed using Image-Pro 6.3 software (Media Cybernetics, Inc, USA). The BCa cell line 5637 and HDF-a were used as positive and negative controls, respectively.

The study was approved by the Ethics Committee of $\mathrm{Xi}$-an Jiaotong University, and each patient gave written informed consent prior to the study.

\section{Fluorescence microscopy images}

Images of cells on glass coverslips were obtained using an Olympus laser scanning confocal fluorescence microscope (FluoView FV1000, Olympus Optical, Tokyo, Japan). For assays using dual FRET MBs, the excitation wavelength was $488 \mathrm{~nm}$, and the emission detection wavelengths were $520 \pm 20$ $\mathrm{nm}$ (FAM donor fluorescence emission wavelength) and $560 \pm 20 \mathrm{~nm}$ (Cy3 acceptor fluorescence emission wavelength), respectively. The signal-to-background ratios were then measured by an Olympus Fluoview Ver.1.6a Viewer (Olympus Optical, Tokyo, Japan).

Fluorescence images were acquired using an Olympus fluorescence microscope. The excitation filter range was 470-490 $\mathrm{nm}$, and the emission filter range was $580 \mathrm{~nm}$ long-pass interference filters (IF). The signal-to-background ratios were measured using Image-Pro 6.3 software at the same instrument settings used for acquisition.

\section{Statistical analysis}

All statistical analyses were performed using SPSS 15.0 (SPSS Inc, Chicago, IL, USA). Quantitative data are presented as the mean \pm SE. The differences in signal-to-background ratios of different combinations of cells and MBs were compared by one-way ANOVA, followed by Dunnett's $t$ test for separate comparisons. Values of $P<0.05$ were considered statistically significant.

\section{Results}

Solution studies of FRET signal and specificity

In-solution probe-target hybridization studies were carried out to determine the specificity of dual FRET MBs and the pair of dual MBs that had the better FRET effect. As shown in Figure 2A, Cy3-Cy5 dual FRET MBs and different MBs were detected with synthetic survivin targets. These results implied that even when a large amount of a single (donor or acceptor) MB was present, the resulting fluorescence at the FRET detection wavelength of $665 \mathrm{~nm}$ (purple curve) was still much lower than the FRET signal when both were present (blue curve). The FAM-Cy3 dual FRET MB pair had the same fluorescence signal at $515 \mathrm{~nm}$ and $565 \mathrm{~nm}$ (blue curve), which implied a higher FRET efficiency than the Cy3-Cy5 dual FRET MB pair (Figure 2B). Thus, the FAM-Cy3 dual FRET MB pair was chosen for further studies.

\section{Western blot analysis of survivin protein expression}

Western blot analyses were performed to detect the protein level of survivin in human BCa cell lines and HDF-a cells. Using GAPDH as the loading control, we found that survivin was expressed in human BCa cell lines (5637, 253J, and T24 cells) but was not expressed in HDF-a cells (Figure 2C).

\section{Survivin mRNA detection in BCa cells}

Our previous study showed that survivin mRNA could be detected in a BCa cell line using survivin $\mathrm{MBs}^{[7,8]}$. The HDF-a cells were used as a negative control because they do not 
A

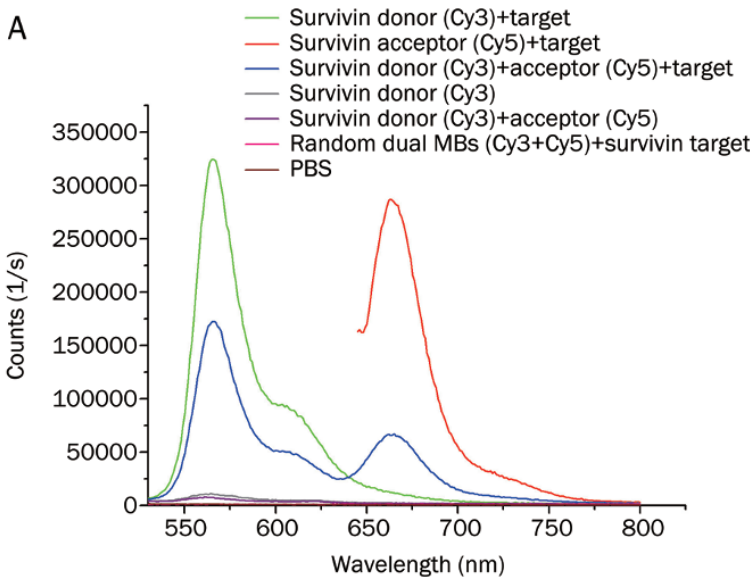

B

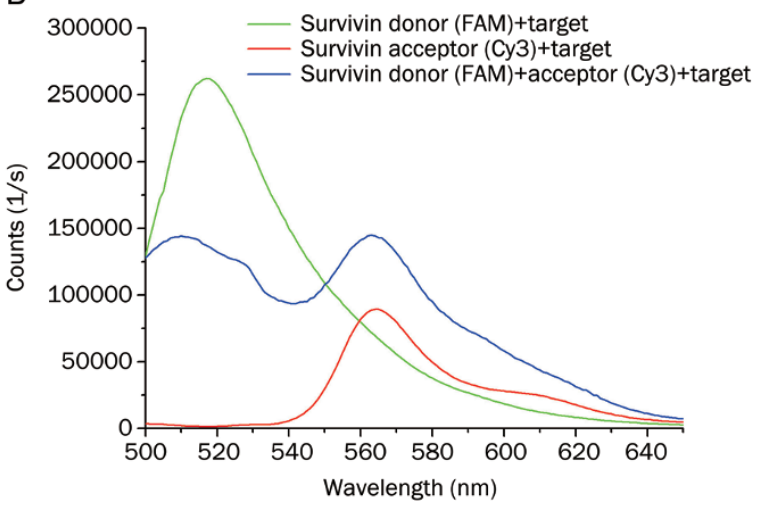

C

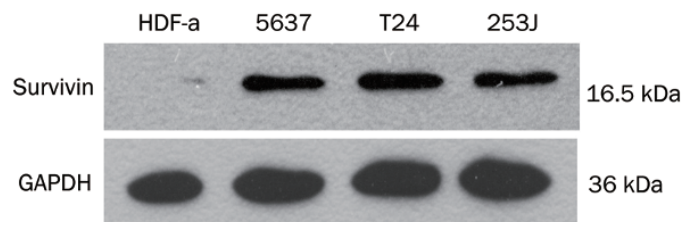

Figure 2. (A) Solution studies of probe-target hybridization of dual FRET MBs. Fluorescence emission spectra of the Cy3 donor and Cy5 acceptor. The green curve was generated from the MB donor in the presence of the target (515 nm excitation, Сy3 second peak excitation). The red curve was generated from the MB acceptor in the presence of the target (645 $\mathrm{nm}$ excitation, Cy5 excitation). The blue curve was generated from both donor and acceptor MBs in the presence of the target ( $515 \mathrm{~nm}$ excitation). The gray curve was generated from the MB donor in the absence of the target (515 $\mathrm{nm}$ excitation). The purple curve was generated from both donor and acceptor MBs in the absence of the target (515 nm excitation). The pink curve was generated from random dual MBs in the presence of the survivin target (515 nm excitation). The wine-colored curve was generated from PBS. (B) Solution studies of probe-target hybridization of dual FRET MBs. Fluorescence emission spectra of the FAM donor and the Cy3 acceptor under FAM excitation at $490 \mathrm{~nm}$. The green curve was generated from the MB donor in the presence of the target. The red curve was generated from the MB acceptor in the presence of the target. The blue curve was generated from both donor and acceptor MBs in the presence of the target. (C) Western blot analyses of survivin protein expression in BCa cell lines (5637, T24, and 253J) and the normal cell line, HDF-a. GAPDH was the loading control. express survivin mRNA ${ }^{[11,12]}$. In this study, survivin dual FRET MBs, the donor and acceptor beacons, were delivered into 5637 cells and HDF-a cells, respectively. Figure 3A shows the laser scanning confocal fluorescence microscopy images of 5637 cells and HDF-a cells irradiated with $488 \mathrm{~nm}$ (the FAM donor fluorescence excitation wavelength). In cells incubated only with survivin dual FRET MBs, the 5637 cells exhibited much stronger fluorescence at $560 \pm 20 \mathrm{~nm}$ (the Cy3 acceptor fluorescence emission wavelength) than at $520 \pm 20 \mathrm{~nm}$ (the FAM donor fluorescence emission wavelength), indicating an excellent FRET efficiency. At the same time, almost no fluorescence was detected in HDF-a cells incubated with survivin dual FRET MBs, and only weak fluorescence was detected in 5637 cells incubated with donor beacon and acceptor beacon, respectively.

Because laser scanning confocal fluorescence microscopy is expensive and is not routinely used in clinical settings, we also tried to detect FRET signals using ordinary fluorescence microscopy. The excitation filter range was $470-490 \mathrm{~nm}$, and the emission filter range was $580 \mathrm{~nm}$ long-pass IF. As shown in Figure 3B, almost no fluorescence signal was detected in HDF-a cells incubated with dual FRET MBs or in 5637 cells incubated with donor MBs and acceptor MBs, respectively. The signal-to-background ratios of HDF-a cells and 5637 cells incubated with donor MBs or acceptor MBs were 1.1, 1.5, and 1.2 , respectively, but obvious fluorescence signal was detected in 5637 cells incubated with dual FRET MBs, with a signalto-background ratio of 5.8 (Figure 3D). These results demonstrated that ordinary fluorescence microscopy could be used for the detection of survivin mRNA in BCa cells using dual FRET MBs.

Using ordinary fluorescence microscopy, we also evaluated FRET signals in other BCa cell lines (253J and T24) and in exfoliated cells from the urine of healthy adults and patients with BCa. As shown in Figure 4, significant fluorescence was detected in 253J and T24 cells and in exfoliated cells from the urine of patients with BCa; however, fluorescence was not detected in exfoliated cells from the urine of healthy adults.

\section{Discussion}

Currently, cystoscopy and cytology remain the gold standard for BCa detection and follow-up. However, conventional cystoscopy is invasive, expensive, and time-consuming. Moreover, several complications can occur after cystoscopy. Additionally, cystoscopy is associated with patient discomfort and is thus refused in some cases ${ }^{[6]}$. Although urinary cytology has been accepted as the most reliable technique for the monitoring of non-invasive $\mathrm{BCa}$, it lacks adequate sensitivity, particularly for the detection of the low-grade tumors ${ }^{[6,14]}$. The development of new non-invasive methods of detection for tumor markers that can reliably predict the presence of a tumor would be clinically important in terms of disease management, quality of life and care cost. However, none of the currently available diagnostic markers can replace cystoscopy ${ }^{[6]}$. 
A
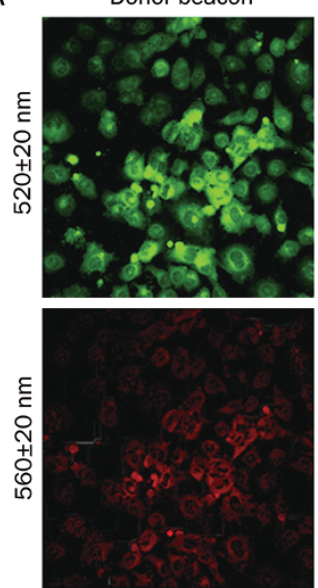

5637

B

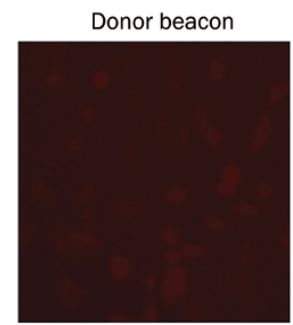

5637
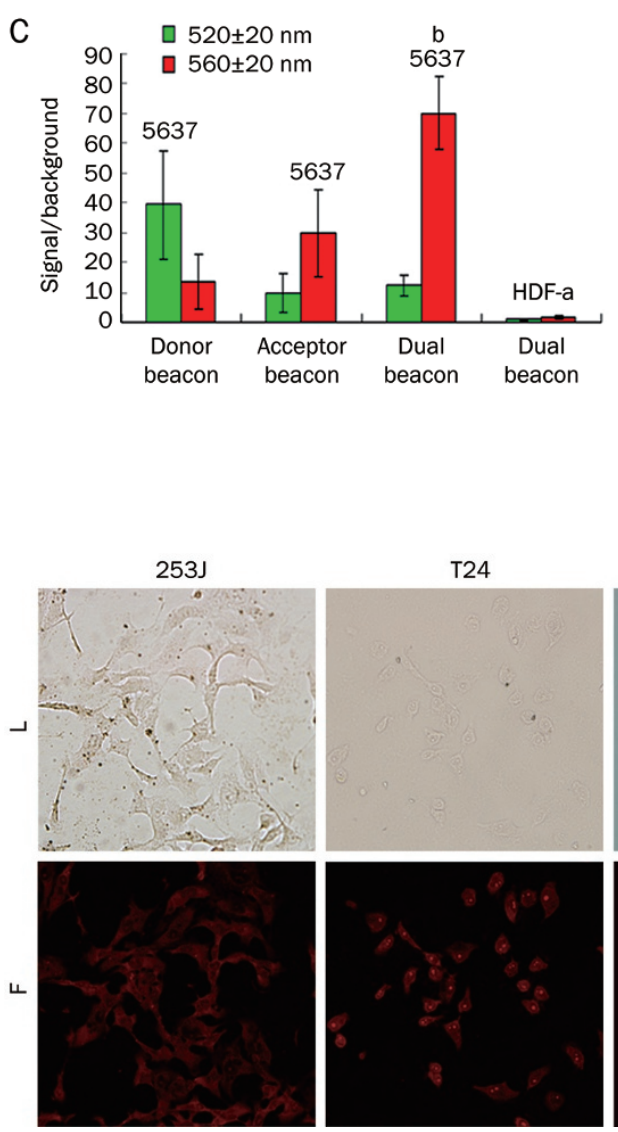
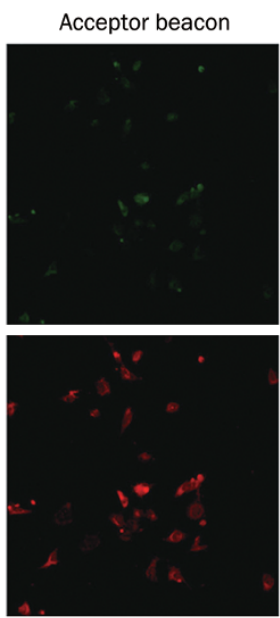

5637

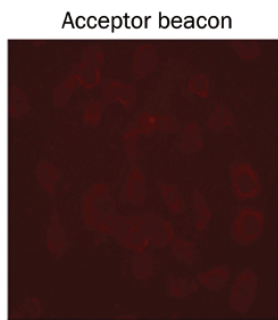

5637

D

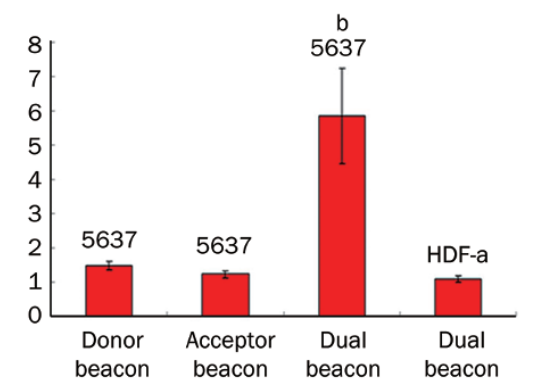

Dual beacon
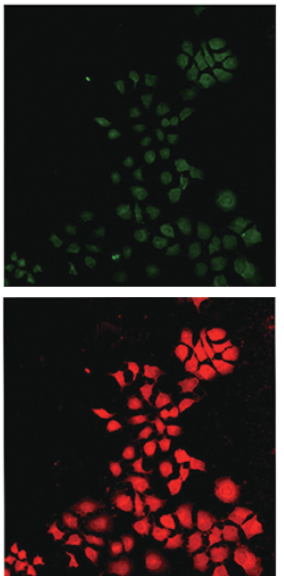

5637

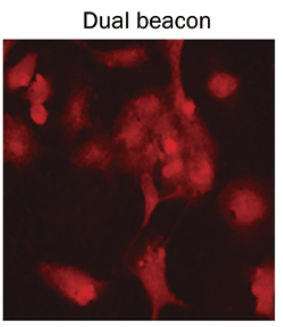

5637

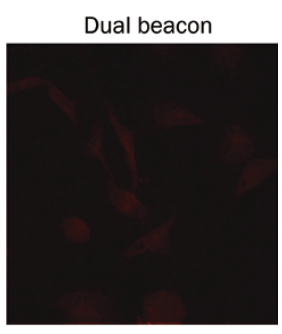

HDF-a
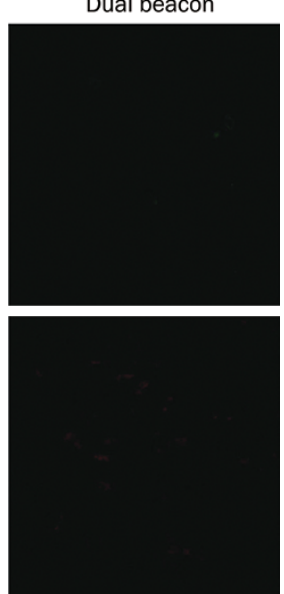

HDF-a

Dual beacon confocal fluorescence microscopy images of 5637 cells and HDF-a cells. At an emission wavelength of $560 \pm 20 \mathrm{~nm}$, the 5637 cells incubated with dual FRET MBs gave a much better signal-to-background ratio than other groups $\left({ }^{b} P<0.05\right)$. (D) The signal-to-background ratios of fluorescence microscopy images of 5637 cells and HDF-a cells. The 5637 cells incubated with FRET MBs gave a much better signal-to-background ratio than other groups $\left({ }^{\mathrm{b}} \mathrm{P}<0.05\right)$.

Figure 4. Survivin mRNA detection in BCa cell lines and exfoliated cells in urine from patients with $\mathrm{BCa}$ and healthy adults using fluorescence microscopy. Significant fluorescence signal was detected in 253J and T24 cells, and exfoliated cells from the urine of patients with $\mathrm{BCa}$ but not in exfoliated cells from the urine of healthy adults. $L=$ light field, $F=$ red fluorescence field (magnification $\times 200$ ). 
Survivin, a member of the IAP family, has significantly higher expression in urothelial tumors than in normal tissue $^{[15]}$. Survivin mRNA in the urine of patients with BCa has been considered to be a potentially useful urinary marker with diagnostic value $\mathrm{e}^{[16,17]}$.

Our previous studies applied survivin MBs to detect BCa cells in urine samples, and they showed that MBs are sensitive and simple to use. Although our results held great promise for the diagnosis and follow-up of patients with $\mathrm{BCa}^{[7,8]}$, the specificity of the survivin MBs was inadequate, and a number of patients with nonurothelial tumors tested positive with the $\mathrm{MB}$ assay ${ }^{[7,8]}$. This was partly due to the degradation of conventional MBs by nucleases and to the MBs being opened by nucleic acid binding proteins, which led to false positive signals ${ }^{[9]}$. As a result of the low background in the dual FRET $\mathrm{MB}$ approach ${ }^{[11]}$, improved detection specificity compared with MBs was achieved. Thus, we designed and synthesized survivin dual FRET MBs to reduce false positive signals while maintaining the advantages of convenience and speed.

The FRET probe pair consisted of two MBs, one labeled with a donor fluorophore (donor beacon) and a second labeled with an acceptor fluorophore (acceptor beacon). These MBs were designed to hybridize to adjacent regions on an mRNA target so that the two fluorophores would lie within the FRET range (about $6 \mathrm{~nm}$ ) when probe/target binding occurred for both beacons. Excitation of the donor fluorophore would then result in fluorescence emission at a wavelength characteristic of the acceptor fluorophore and a positive FRET signal readily differentiable from non-FRET false positive signals that might result from probe degradation and non-specific probe opening $^{[11]}$.

In this study, we investigated the possibility of using survivin dual FRET MBs in the diagnosis of BCa. Because the higher $\mathrm{A} / \mathrm{T}$ ratio adjacent to the survivin $\mathrm{MB}$ target sequence that we previously chose ${ }^{[8]}$ is unsuitable for the formation of the stems of the dual FRET MBs, we chose another survivin target sequence, which was unique and had no overlap with other genes in the IAP family ${ }^{[11]}$. The assays of survivin dual FRET MB probe-target hybridization in solution indicated that the FRET signal was much higher than the non-FRET signal, and the FAM-Cy3 dual FRET MB pair had a higher FRET efficiency than Cy3-Cy5 dual FRET MB pair (Figure 2). This was due to the larger overlap between the emission spectrum of FAM (donor fluorophore) and the excitation spectrum of Cy3 (acceptor fluorophore), which allowed a more efficient energy transfer than with the Cy3-Cy5 pair ${ }^{[18]}$. Thus, in our subsequent studies, FAM and Cy3 were used as the donor and acceptor fluorophores, respectively.

The human BCa cell line 5637, which has been reported to have moderate expression of $\operatorname{survivin}^{[7,8,19]}$, was used in our previous study ${ }^{[7,8]}$ and in the present investigation. The laser scanning confocal fluorescence microscopy images (Figure 3) show that the emission at $560 \pm 20 \mathrm{~nm}$ (the Cy3 acceptor fluorescence emission wavelength) results in a stronger fluorescence signal than the emission at $520 \pm 20 \mathrm{~nm}$ (the FAM donor fluorescence emission wavelength), indicating the excellent specificity of the dual FRET MBs.

Laser scanning confocal fluorescence microscopy is expensive and is not routinely used in clinical settings compared with fluorescence microscopy. Based on the widespread use and availability of fluorescence microscopy in clinical settings, we investigated the possibility of using dual MBs to detect $\mathrm{BCa}$ with a fluorescence microscope. The fluorescence signal could only be detected in BCa cells incubated with dual FRET MBs. To the best of our knowledge, this is the first report of the detection of BCa using dual FRET MBs with either confocal fluorescence microscopy or ordinary fluorescence microscopy. Additionally, a much better signal-to-background ratio was obtained with the dual FRET MBs than with the conventional MBs, indicating a high level of specificity of the dual FRET MBs. Similar effects were also observed with lung cancer, breast cancer, gastric cancer and cervical cancer cell lines (unpublished data), suggesting the potential use of dual FRET MBs in the detection of many different cancers. Moreover, the time required for survivin dual FRET MB detection was 2-3 $h$ in our study. This clearly demonstrates that the dual FRET $\mathrm{MB}$ approach is easy to perform and is specific for the detection of survivin mRNA levels in BCa cells.

In conclusion, this study demonstrates that survivin dual FRET MBs provide a simple, fast and non-invasive approach with excellent specificity for the detection of survivin mRNA in BCa cells. The use of dual FRET MBs for the detection of survivin mRNA in urinary exfoliated cells from patients with $\mathrm{BC}$ is under investigation.

\section{Acknowledgements}

We thank Prof Xiao-hong FANG from the Institute of Chemistry, Chinese Academy of Sciences, for her helpful support. This work was supported by the National Natural Science Foundation of China (№ 30672102).

\section{Author contribution}

Zhi-qiang WANG, Jun ZHAO, Luke S CHANG, and Da-lin HE designed the research; Zhi-qiang WANG, Jun ZHAO, Xin-yang WANG, Jin ZENG, Kai-jie WU, and Yu-le CHEN performed the research; Zhi-qiang WANG, Jun ZHAO, and Jin ZENG analyzed the data; and Zhi-qiang WANG and Jin ZENG wrote the paper.

\section{References}

1 Jemal A, Siegel R, Ward E, Hao Y, Xu J, Thun MJ. Cancer statistics, 2009. CA Cancer J Clin 2009; 59: 225-49.

2 Sasaki T, Horikawa M, Orikasa K, Sato M, Arai Y, Mitachi Y, et al. Possible relationship between the risk of Japanese bladder cancer cases and the CYP4B1 genotype. Jpn J Clin Oncol 2008; 38: 634-40.

3 Tsui $\mathrm{KH}$, Juang $\mathrm{HH}$, Lee TH, Chang PL, Chen CL, Yung BY. Association of nucleophosmin/B23 with bladder cancer recurrence based on immunohistochemical assessment in clinical samples. Acta Pharmacol Sin 2008; 29: 364-70.

4 Van Tilborg AA, Bangma CH, Zwarthoff EC. Bladder cancer biomarkers and their role in surveillance and screening. Int J Urol 2009; 16: 23-30.

5 Schultz IJ, Witjes JA, Swinkels DW, de Kok JB. Bladder cancer 
diagnosis and recurrence prognosis: comparison of markers with emphasis on survivin. Clin Chim Acta 2006; 368: 20-32.

6 Van Rhijn BW. Considerations on the use of urine markers for bladder cancer. Eur Urol 2008; 53: 880-1.

7 Zhao J, He D, He H, Li L, Zhang LL, Wang XY. Primary application study in early diagnosis of bladder cancer by survivin molecular beacons. Urology 2007; 70: 60-4.

8 Zhao J, Wang ZQ, Wang XY, Yang XJ, He D. Preliminary study of diagnostic utility of molecular beacons in bladder cancer. Urology 2010; 76: 512-8.

9 Chen AK, Behlke MA, Tsourkas A. Avoiding false-positive signals with nuclease-vulnerable molecular beacons in single living cells. Nucleic Acids Res 2007; 35: e105.

10 Krusinski T, Ozyhar A, Dobryszycki P. Dual FRET assay for detecting receptor protein interaction with DNA. Nucleic Acids Res 2010; 38: e108.

11 Santangelo PJ, Nix B, Tsourkas A, Bao G. Dual FRET molecular beacons for mRNA detection in living cells. Nucleic Acids Res 2004; 32: e57.

12 Yang L, Cao Z, Lin Y, Wood WC, Staley CA. Molecular beacon imaging of tumor marker gene expression in pancreatic cancer cells. Cancer Biol Ther 2005; 4: 561-70.
13 Wu KJ, Zeng J, Zhu GD, Zhang LL, Zhang D, Li L, et al. Silibinin inhibits prostate cancer invasion, motility and migration by suppressing vimentin and MMP-2 expression. Acta Pharmacol Sin 2009; 30: 1162-8.

14 Lekili M, Sener E, Demir MA, Temeltas G, Muezzinoglu T, Buyuksu C. Comparison of the nuclear matrix protein 22 with voided urine cytology in the diagnosis of transitional cell carcinoma of the bladder. Urol Res 2004; 32: 124-8.

15 Liu L, Zhang M, Zou P. Expression of PLK1 and survivin in nonHodgkin's lymphoma treated with CHOP. Acta Pharmacol Sin 2008; 29: 371-5.

16 Vrooman OP, Witjes JA. Urinary markers in bladder cancer. Eur Urol 2008; 53: 909-16.

17 Weikert S, Christoph F, Schrader M, Krause H, Miller K, Muller M. Quantitative analysis of survivin mRNA expression in urine and tumor tissue of bladder cancer patients and its potential relevance for disease detection and prognosis. Int J Cancer 2005; 116: 100-4.

18 Wang $\mathrm{Y}$, Wang N. FRET and mechanobiology. Integr Biol (Camb) 2009; 1: 565-73.

19 Ning S, Fuessel S, Kotzsch M, Kraemer K, Kappler M, Schmidt U, et al. siRNA-mediated down-regulation of survivin inhibits bladder cancer cell growth. Int J Oncol 2004; 25: 1065-71. 